\title{
BMJ Open Protocol of the Australasian Malignant Pleural Effusion-2 (AMPLE-2) trial: a multicentre randomised study of aggressive versus symptom-guided drainage via indwelling pleural catheters
}

To cite: Azzopardi M, Thomas R, Muruganandan S, et al. Protocol of the Australasian Malignant Pleural Effusion-2 (AMPLE-2) trial: a multicentre randomised study of aggressive versus symptomguided drainage via indwelling pleural catheters. BMJ Open 2016;6: 011480 . doi:10.1136/bmjopen-2016011480

- Prepublication history and additional material is available. To view please visit the journal (http://dx.doi.org/ 10.1136/bmjopen-2016011480).

Received 11 February 2016 Revised 7 May 2016 Accepted 8 June 2016

\section{CrossMark}

For numbered affiliations see end of article.

Correspondence to Professor Y C Gary Lee; gary.lee@uwa.edu.au

\section{ABSTRACT}

Introduction: Malignant pleural effusions (MPEs) can complicate most cancers, causing dyspnoea and impairing quality of life (QoL). Indwelling pleural catheters (IPCs) are a novel management approach allowing ambulatory fluid drainage and are increasingly used as an alternative to pleurodesis. IPC drainage approaches vary greatly between centres. Some advocate aggressive (usually daily) removal of fluid to provide best symptom control and chance of spontaneous pleurodesis. Daily drainages however demand considerably more resources and may increase risks of complications. Others believe that MPE care is palliative and drainage should be performed only when patients become symptomatic (often weekly to monthly). Identifying the best drainage approach will optimise patient care and healthcare resource utilisation.

Methods and analysis: A multicentre, open-label randomised trial. Patients with MPE will be randomised 1:1 to daily or symptom-guided drainage regimes after IPC insertion. Patient allocation to groups will be stratified for the cancer type (mesothelioma vs others), performance status (Eastern Cooperative Oncology Group status $0-1$ vs $\geq 2$ ), presence of trapped lung (vs not) and prior pleurodesis (vs not). The primary outcome is the mean daily dyspnoea score, measured by a $100 \mathrm{~mm}$ visual analogue scale (VAS) over the first 60 days. Secondary outcomes include benefits on physical activity levels, rate of spontaneous pleurodesis, complications, hospital admission days, healthcare costs and QoL measures. Enrolment of 86 participants will detect a mean difference of VAS score of $14 \mathrm{~mm}$ between the treatment arms (5\% significance, $90 \%$ power) assuming a common

\section{Strengths and limitations of this study}

Multicentre randomised trial comparing aggressive (daily) versus symptom-guided drainage regimes after indwelling pleural catheter (IPC) insertion for patients with malignant pleura effusions.

- Variations in drainage approaches have direct impact on patient care and major implications on healthcare resources and costs. Establishing the optimal approach will impact clinical care.

- The trial builds on the infrastructure and recruitment network established via the recently completed Australasian Malignant Pleural Effusion-1 (AMPLE-1) study.

between-group SD of $18.9 \mathrm{~mm}$ and a $10 \%$ lost to follow-up rate.

Ethics and dissemination: The Sir Charles Gairdner Group Human Research Ethics Committee has approved the study (number 2015-043). Results will be published in peer-reviewed journals and presented at scientific meetings.

Trial registration number: ACTRN12615000963527; Pre-results.

\section{INTRODUCTION}

Over 8000 Australians and 1 million patients worldwide develop a malignant pleural effusion (MPE) each year. MPE can complicate most cancers, including $30 \%$ of lung and breast carcinomas and over $90 \%$ of patients 
with malignant pleural mesothelioma. ${ }^{1}$ Breathlessness is the most common symptom of MPE and is often disabling and significantly impairs daily activities and quality of life (QoL). The development of an MPE generally heralds an incurable cancer with a median survival of 4-12 months for metastatic pleural carcinomas and mesotheliomas, respectively. ${ }^{2}$ Conventional therapies are suboptimal and patients often have to undergo multiple pleural interventional procedures for fluid drainage. These procedures are painful, costly, can be associated with major complications and require medical visits or hospitalisations, depriving patients of precious time in their limited lifespan. ${ }^{3}$ Talc pleurodesis, a therapy first described in $1935,{ }^{4}$ remains the most commonly used definitive therapy worldwide; however, data in recent years have shown that this approach has high failure rates, ${ }^{5}$ involves more time in hospital ${ }^{6}$ and is associated with significant side effects. ${ }^{7}$

Indwelling pleural catheter (IPC) is a novel treatment for MPE that has considerable advantages over talc pleurodesis, providing equal benefits, as first-line treatment, in the relief of breathlessness and chest pain, ${ }^{8}$ and offers at least as good improvements in QoL. IPC can be applied to most patients with MPE, whereas pleurodesis is only suitable in those with fully expanded lungs. Significantly fewer patients treated with an IPC require subsequent pleural drainages in their remaining lifespan than those pleurodesed. ${ }^{5-11}$ In two randomised trials, patients in the IPC group spent significantly shorter time in hospital than those receiving pleurodesis for the initial procedure; ${ }^{811}$ reduction of inpatient stay can represent significant savings on healthcare costs. Spontaneous pleurodesis can develop in patients fitted with an IPC, thus allowing removal of the IPC and reducing further drainage costs and adverse events. Recent studies have shown that serious IPC complications are uncommon, with a $4.7 \%$ incidence of pleural infection ${ }^{6}$ and $10 \%$ incidence of catheter tract metastases. ${ }^{12}$

Despite the reported benefits of IPC, its use is relatively new and significant heterogeneity exists on practical aspects of IPC management around the world. Opinions are polarised even among world experts in the optimal approach to fluid drainage regimes because of lack of quality data on the subject. The variations in drainage approaches have direct impact on patient care and major implications on healthcare resources and costs.

\section{Aggressive versus symptom-guided drainages}

One school advocates aggressive removal of fluid, usually by daily or alternate-day drainage via the IPC. This approach is the standard practice in many medical centres in the USA and Canada. On the other hand, many centres in the UK believe that MPE care should be palliative and symptom based. Hence, fluid drainage should only be performed if and when patients become symptomatic from MPE accumulation. Drainage is typically performed from weekly to monthly according to individual needs.
Those who advocate 'aggressive drainage' argue that daily fluid evacuation allows the best control of symptoms and thus maximal exercise and physical activities. It is believed that keeping the pleural cavity dry permits approximation of the visceral and parietal pleura and promotes their symphysis. The resultant spontaneous pleurodesis will allow removal of the IPC and reduce long-term costs and complication rates. Reported rates of spontaneous pleurodesis have varied; ${ }^{13}$ Tremblay et $a l^{14}$ reported that up to $70 \%$ of those who did not have trapped lungs will develop a spontaneous pleurodesis, whereas in another study of all MPE patients treated with an IPC, a spontaneous pleurodesis rate of $45 \%$ (range: $26-57 \%$ ) with a mean time to pleurodesis of 52 days ${ }^{15}$ was reported. Once spontaneous pleurodesis is achieved, few $(<7.9 \%)$ required further pleural intervention. ${ }^{14} 15$

Those who advocate 'symptom-guided drainage' argue that fluid drainage in the absence of breathlessness is unlikely to improve well-being or QoL. Daily drainages involve considerably more time, effort on the part of the patient and their caregivers and consumable costs. For instance, daily drainage will cost 14 times more in time and consumable costs compared with those who drain fortnightly. Frequent access of the IPC may theoretically increase the risks of complication-for example, infection.

\section{METHODS AND ANALYSIS}

The Australian Malignant PLeural Effusion (AMPLE) trial-2 is a multicentre, open-label randomised (trial entry) study designed to define if either of the two common drainage regimes (aggressive or symptomguided drainage) is superior to the other in improving patient-relevant clinical outcomes, especially breathlessness, and their safety. The nature of the intervention means that investigators and patients cannot be blinded to the treatment allocation.

\section{Primary end point}

The degree of breathlessness will be assessed using a $100 \mathrm{~mm}$ visual analogue scale (VAS) score, recorded daily in the first 60 days. This is the most validated measure of dyspnoea in MPE and was successfully used in MPE trials-for example, Second Therapeutic Intervention in Malignant Effusion Trial (TIME-2) ${ }^{8}$ $(\mathrm{n}=106)$ and AMPLE-1 $(\mathrm{n}=146) .{ }^{16}$

\section{Secondary end points}

Secondary end points include other clinical outcomes of major relevance to patients with MPE. Physical activity patterns will be evaluated by a well-validated triaxial accelerometer (ActiGraph GT3X+). QoL will be measured by EQ-5D-5L and a $100 \mathrm{~mm}$ EQ-Visual Analogue Scale (EQ-VAS). The rate of spontaneous pleurodesis, hospitalisation, adverse events and healthcare costs will be compared. 


\section{SETTING}

The trial will include 86 participants with MPEs randomised 1:1 to either the aggressive (daily) or symptomguided drainage regimes after IPC insertion (figure 1). Trial details are as per AMPLE-2 trial protocol V.1 (date 25 March 2015).

\section{POWER CALCULATION}

The study will involve centres in Australia, New Zealand, Hong Kong and Malaysia, most of which are teaching hospitals. Enrolment of 86 participants will detect a mean difference of VAS score of $14 \mathrm{~mm}$ between the treatment arms (5\% significance, $90 \%$ power) assuming a common between-group SD of $18.9 \mathrm{~mm}$ (based on the TIME-2 trial $^{8}$ ) and a $10 \%$ lost to follow-up rate. The minimal clinically important difference for the VAS score in this setting is $19 \mathrm{~mm}$ ( $95 \%$ CI 14 to $24 \mathrm{~mm}$ ) as per Mishra et $a .^{17}$ The lower end of the CI of $14 \mathrm{~mm}$ was used for this power calculation.

\section{STATISTICAL PLAN}

Data will be analysed on an intention-to-treat basis. The difference between the two groups will be estimated using a linear mixed-effects model, taking into account the repeated VAS scores measured on the same participant, and missing VAS scores (assuming that missing scores are missing at random). This model will be adjusted for the minimisation variables. A secondary analysis, again using linear mixed-effects models, will investigate the effect of time from randomisation and will include random intercepts and time effects as appropriate, in addition to time and treatment interaction terms.

The economic evaluation will be a cost-effectiveness analysis comparing differences in costs and qualityadjusted life years (QALYs) between the two groups over the duration of the trial. EQ-5D-5L response scores will be converted into utility values using an algorithm for the Australian population, ${ }^{18}$ with QALYs calculated from utility values. The perspective adopted for the evaluation will be the health sector. Simple regression analysis using the measured covariates will be conducted to guide the extent to which more complex analysis can be undertaken to take account of the distributional characteristics of the data and any differences in baseline characteristics despite random allocation. Incremental cost-effectiveness ratios will be calculated as the difference in the cost of drainage regimes divided by the difference in QALYs.

\section{PARTICIPANT SCREENING AND SELECTION}

All patients who require IPC placement for management of an MPE will be identified by the principal investigator (PI) of each study site. Screening criteria are based on standard clinical practice, and consecutive eligible patients will be offered trial entry. The PI or a nominated member of staff will approach participants who fulfil the criteria to enter the trial. Screening logs will be kept.

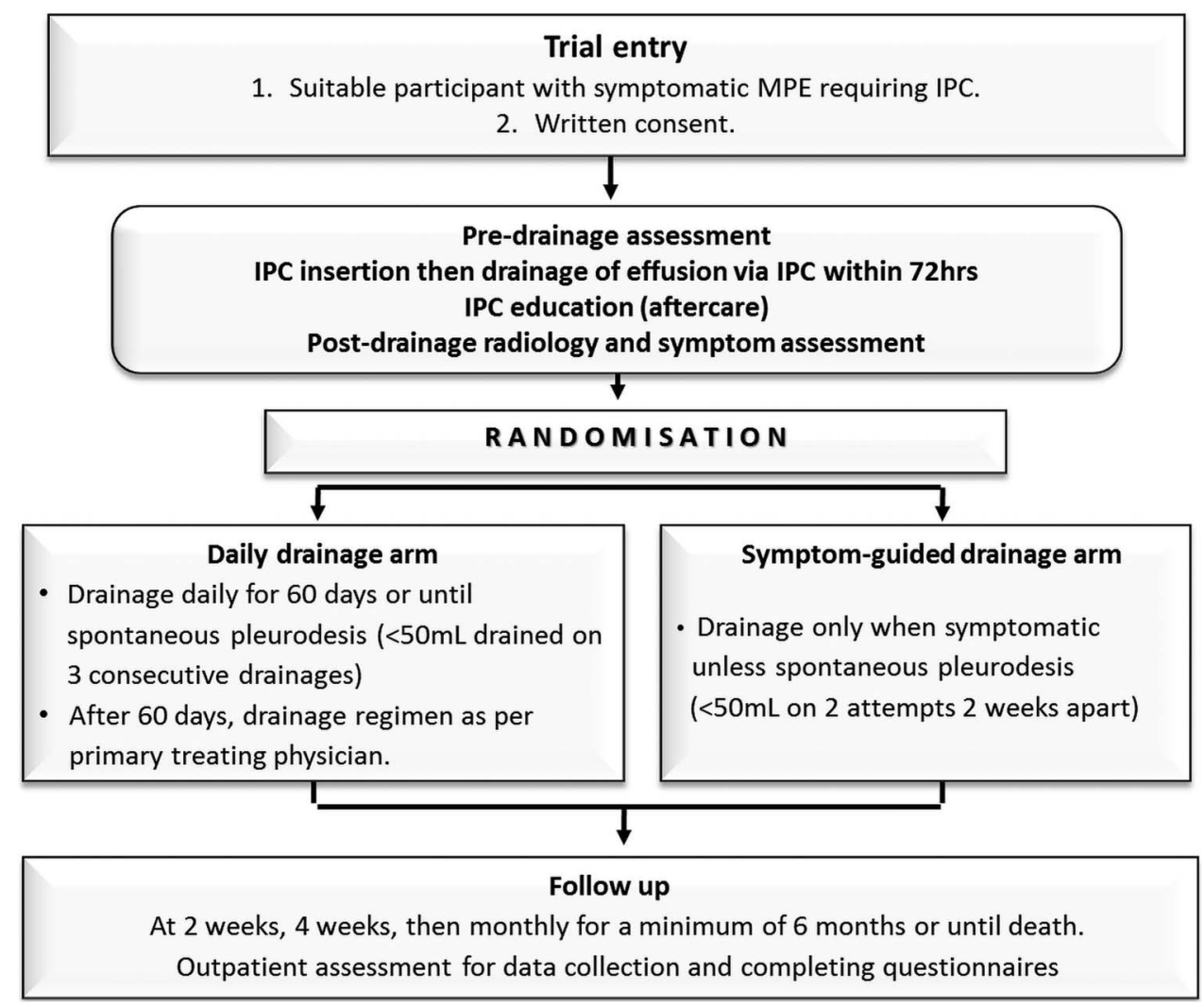

Figure 1 Study flow chart. IPC, indwelling pleural catheter; MPE, malignant pleural effusion. 


\section{Inclusion criteria}

Patients with a symptomatic MPE in whom fluid drainage via an IPC is considered appropriate by the managing clinician. An MPE is defined as one in which malignant cells are identified in the pleural fluid or pleural biopsy; or a large exudative pleural effusion without other causes in a patient with known disseminated extrathoracic malignancy.

\section{Exclusion criteria}

Age <18 years; expected survival $<3$ months; pleural infection; chylothorax; pregnancy or lactation; uncorrectable bleeding diathesis; previous ipsilateral lobectomy/pneumonectomy; significant loculations likely to preclude effective fluid drainage; significant visual impairment and inability to consent or comply with the protocol.

\section{INFORMED CONSENT}

The potential participant will be approached by the PI or nominated investigator about the possibility of taking part in the study once they have decided to be treated with an IPC to manage their MPE. They will be given an explanation of the study by the doctor and the Participant Information and Consent Form. The patient can either provide informed consent while at the clinic or during the inpatient consultation or be given the option to discuss the study with family, friends and general practitioner (GP) before making a decision. They can discuss the study further with the study team when they return for their next clinic appointment or hospital admission for insertion of their IPC.

\section{RANDOMISATION}

Participants will be randomly assigned (1:1) to either aggressive or symptom-guided drainage via their IPC. Randomisation will include minimisation for the following:

1. Cancer type (mesothelioma vs non-mesothelioma);

2. Performance status (Eastern Cooperative Oncology Group (ECOG) $0-1$ vs $\geq 2$ );

3. Presence of trapped lung-see online supplementary appendix 1 -(vs not);

4. Prior pleurodesis (vs not).

Recent publications have identified cancer type and ECOG status as major predictors of survival in MPE. ${ }^{2}$ The presence of trapped lung and prior failed pleurodesis are known factors that reduce the likelihood for spontaneous pleurodesis and thus impact on many of the clinical outcome measures. There will be no stratification by centre. The National Health and Medical Research Council (NHMRC) Clinical Trials Centre will provide the randomisation set-up using their automated telephone-based interactive voice response service.

\section{STANDARD CARE}

Irrespective of the drainage strategy the participant is randomised to, all participants and carers are given standard education (including information sheet and educational video materials where appropriate) and advice on drainage method, IPC aftercare and potential complications on the routine care of the IPC. Participants and their carers have the support and care of the respiratory community nurses as per standard care and can readily access support services (eg, via direct phone line) if any concern arises. The local PI will be responsible to ensure only properly trained clinical doctors and health staff are involved in the procedures and ongoing aftercare of the IPC. The IPC can be removed if clinically indicated (eg, spontaneous pleurodesis).

All the participants will be managed by their own clinical teams and receive all other medical treatments (including chemotherapy and radiotherapy, if appropriate) as per standard participant care.

\section{INTERVENTIONS}

\section{The aggressive drainage arm}

Participants will drain their MPE via their IPC every day for the first 60 days unless:

A. Clinically contraindicated or

B. Spontaneous pleurodesis occurs. This is defined as $<50 \mathrm{~mL}$ of fluid removed on three consecutive drainages, in the absence of any significant residual pleural fluid collections on imaging.

\section{The symptomatic drainage arm}

Participants will drain their MPE via their IPC only if they feel the effusion-related symptoms (usually breathlessness, cough and/or tightness) have recurred or at least once per fortnight. In the symptom-guided arm, spontaneous pleurodesis is defined as $<50 \mathrm{~mL}$ drainage in two attempts 2 weeks apart, in the absence of significant residual fluid on imaging.

The intervention, that is, the drainage frequency, can be modified if clinically indicated as judged by the patients' attending physician.

\section{DATA COLLECTION AND MANAGEMENT}

Clinical data, baseline VAS scores for breathlessness and QoL (VAS and EQ-5D-5L) measure will be collected prior to IPC insertion and at 72 hours postinsertion (see online supplementary file: AMPLE2 SPIRIT Schedule). Chest radiographs will be assessed for effusion size and the presence of trapped lung and fluid loculations prior to IPC insertion and at 72 hours postinsertion. Participants will be asked to keep a logbook recording their VAS score for breathlessness, measured daily for 60 days, then weekly until the end of the study or death. Participants will also record fluid drainage volumes for each drainage and mark their pain level on the VAS pain scale. Participants will be reviewed at 2 and 4 weeks, and thereafter monthly for a minimum of 6 months and 
data collected on secondary end points. Physical activity levels as measured by the 7-day triaxial accelerometer will be assessed at sites where the device is available. Actigraphy will be applied for 1 week at randomisation and thereafter 1 week each month for a minimum of 6 months. Hospital admission details including length of stay and any adverse events and their management will be recorded.

Data will be entered into a secure study database, and a system of data validation checks will be implemented and applied to the database. The accuracy of the data will be verified by comparing study data to source documents.

\section{PRIMARY OUTCOME}

The degree of breathlessness will be assessed using a $100 \mathrm{~mm}$ VAS score, recorded daily in the first 60 days, then weekly until the end of the study or death. The VAS is a $100 \mathrm{~mm}$ line anchored with 'no breathlessness' at $0 \mathrm{~mm}$ and 'worst breathlessness imaginable' at $100 \mathrm{~mm}$. Participants are asked to make a mark along the line at a point representing their level of breathlessness over the preceding 24 hours and at the same time each day. All VAS scores will be measured by two independent researchers and the mean score calculated. If the two measurements differ by $>3 \mathrm{~mm}$, both measurements will be repeated by the same observers.

\section{SECONDARY OUTCOMES}

\section{Hospital admission}

Hospitalisation for any causes (except for elective admissions for chemotherapy) will be recorded for all participants postrandomisation until the end of follow-up period or death, whichever comes first. Admission will be analysed as total admission days (and number of episodes) and also as effusion-related admissions. The latter will include all hospitalisations for drainage purposes, for pleural or IPC-related complications such as infection, symptomatic loculation and for removal of the IPC if indicated.

\section{The percentage of participants who achieve spontaneous} pleurodesis

Spontaneous pleurodesis has occurred where there is minimal output in the absence of any significant residual pleural fluid collections, for a period as stated above, which would allow removal of the IPC. This definition has been used in previous trials. ${ }^{19}$

\section{Adverse events}

An adverse event is defined as any complication that is associated with the IPC such as pleural infection, cellulitis, pain, symptomatic loculation, tube blockage, catheter tract metastases, parenchymal air leak, etc.
Quality of life (QoL)

QoL will be assessed using the EQ-5D-5L questionnaire and a $100 \mathrm{~mm}$ VAS. The EQ-5D-5L is a standardised measure of health-related QoL providing a single value for health status. ${ }^{20}{ }^{21}$ The VAS records self-rated health on a straight line, where the end points are labelled 'best quality of life' and 'worst quality of life' imaginable. ${ }^{22}$

\section{Activity levels}

The goals of MPE treatment are to improve patientrelated outcomes such as palliation of breathlessness, pain and ultimately to improve functional levels. Objective physical activity patterns will be assessed by 7-day triaxial accelerometer assessment (ActiGraph GT3X+, Pensacola, Florida, USA) at sites where the device is available.

\section{Health economics}

Data will be captured from local department coding data and community-based costs from patient data. The cost category will be divided into costs of ongoing drainage and adverse events. Resource use associated with the different IPC drainage regimes, including drainage kits and the drainage time by community nurse (if needed), will be obtained from hospital records and patient selfreport, the latter at patient review sessions. Inpatient/ outpatient management of complications will be captured by participant self-report and from hospital records and will include treatments (eg, antibiotics), diagnostic imaging and other interventions related to the adverse events.

\section{Survival}

Survival of all patients will be recorded from date of randomisation to death or end of study follow-up.

\section{ETHICS AND DISSEMINATION}

The trial has been approved (as of 23 January 2016) by the following committees:

1. Sir Charles Gairdner Group Human Research Ethics Committee (HREC) for WA Health hospitals.

2. St John of God Health Care Ethics Committee for Bunbury and Midland Hospital, Western Australia.

3. South Eastern Sydney Local Health District HREC for St George and the Sutherland hospitals, New South Wales; Royal Brisbane and Women's Hospital and Nambour Hospital, Queensland, and Royal Adelaide Hospital, South Australia.

4. UnitingCare Health HREC for Wesley and St Andrew's hospitals, Queensland.

5. Institutional Review Board of the University of Hong Kong/Hospital Authority Hong Kong West Cluster for Queen Mary Hospital, Hong Kong.

Any protocol amendment may result in changes to the patient information and consent form. The investigators must ensure that the amended form receives approval/ favourable opinion from the ethics committee and that it is signed by any patient subsequently entering into the 
trial and those currently in the study, if affected by the amendment.

\section{Safety reporting}

All adverse events relating to the trial procedure occurring during the course of the study will be collected and documented by the investigator according to the specific definitions and instructions detailed in the 'Adverse Event Reporting' section of the Trial Master File. Any serious or significant adverse event, whether or not considered related to the experimental procedure, will be reported as soon as it becomes apparent using the reporting procedure and associated documentation according to local regulatory requirements. Adverse events will be followed up until resolution. Where adverse events are not resolved at study completion, this will be noted on the adverse event log.

\section{Data safety}

All procedures for the handling and analysis of data will be conducted using the Good Clinical Practice (GCP) meeting The International Conference on Harmonisation of Technical Requirements for Registration of Pharmaceuticals for Human Use (ICH) guidelines and the Australian Human Research Ethics Committees, or local equivalent for the handling and analysis of data for clinical trials. Access to the trial dataset will be available to investigators at the lead site only.

\section{Trial monitoring and oversight}

The Trial Steering Committee (TSC) will be responsible for supervision of the trial in its entirety. It will be responsible for ensuring completion of the trial to clinical and ethical standards. Members of the TSC include an independent chairperson, independent member(s), chief investigator and selected investigators, a consumer representative and the trial coordinator. The Data and Safety Monitoring Committee (DSMC) will oversee the monitoring of adverse events and the ethical conduct of the study. The DSMC includes an independent chairperson and two independent members (including a statistician).

\footnotetext{
Author affiliations

${ }^{1}$ Department of Respiratory Medicine, Sir Charles Gairdner Hospital, Perth, Western Australia, Australia

${ }^{2}$ Pleural Medicine Unit, Institute for Respiratory Health, Perth, Western Australia, Australia

${ }^{3}$ Centre for Respiratory Health, School of Medicine \& Pharmacology,

University of Western Australia, Perth, Western Australia, Australia

${ }^{4}$ Department of Medicine, University of Hong Kong, Hong Kong, China

${ }^{5}$ Wesley Hospital, Brisbane, Queensland, Australia

${ }^{6}$ St George and Sutherland Hospital Clinical School, University of New South Wales, Sydney, New South Wales, Australia

${ }^{7}$ Department of Respiratory and Sleep Medicine, Sutherland Hospital, Sydney, New South Wales, Australia

${ }^{8}$ Respiratory Department, Queen Elizabeth Hospital, Ministry of Health Malaysia, Kota Kinabalu, Sabah, Malaysia

${ }^{9}$ The Department of Thoracic Medicine, The Royal Adelaide Hospital, Adelaide, South Australia, Australia

${ }^{10}$ Respiratory Department, Middlemore Hospital, Auckland, New Zealand

${ }^{11}$ Department of Thoracic Medicine, Royal Brisbane and Women's Hospital,

Brisbane, Queensland, Australia
}

${ }^{12}$ Department of Respiratory Medicine, Sunshine Coast Hospital and Health Service, Nambour, Queensland, Australia

${ }^{13}$ Respiratory Department, Royal Perth Hospital, Perth, Western Australia, Australia

${ }^{14}$ Department of Respiratory Medicine, Fiona Stanley Hospital, Perth, Western Australia, Australia

${ }^{15}$ Saint John of God Public and Private Hospital Midland, Midland, Western Australia, Australia

${ }^{16}$ Bunbury Hospital, Western Australian Country Health Service, Bunbury, Western Australia, Australia

${ }^{17}$ Saint John of God Hospital Bunbury, Bunbury, Western Australia, Australia

${ }^{18}$ National Centre for Asbestos Related Diseases, School of Medicine and Pharmacology, University of Western Australia, Perth, Western Australia, Australia

${ }^{19}$ Exercise Medicine Research Institute, Edith Cowan University, Perth, Western Australia, Australia

${ }^{20}$ Institute of Human Performance, The University of Hong Kong, Hong Kong

${ }^{21}$ School of Public Health, Curtin University, Perth, Western Australia,

Australia

${ }^{22}$ Centre for Applied Statistics, University of Western Australia, Perth, Western Australia, Australia

${ }^{23}$ Division of Pulmonary and Critical Care Medicine, Johns Hopkins University, Baltimore, Maryland, USA

${ }^{24}$ Academic Respiratory Unit, School of Clinical Sciences, University of Bristol, Bristol, UK

Contributors YCGL and RT conceived the initial trial concept with advice from NAM and DF-K (cochairs of the Trial Steering Committee). RUN, DH and JC are responsible for designing the accelerometry, health economics and biodiscovery plans, methods and analyses. CAR is the trial manager and oversees the data collection and running of the trial. MA and SM are the trial coordinators. DCLL, LAG, BCHK, MRSRA, PTN, EY, FCH, DF, MB, RS, FP, CCdC and YCGL developed and modified the trial design and protocol to specifics of individual trial centres. RT, YCGL and KM wrote the statistical analysis plan. YCGL is the chief investigator and takes overall responsibility for all aspects of trial design, the protocol and trial conduct. All authors have read and approved the final manuscript.

Funding This study is supported by project grant funding from the Cancer Council of Western Australia and the Sir Charles Gairdner Research Advisory Group. Rocket Medical (UK) PIc provided the drainage kits without charge for the participants of the study and an unrestricted educational grant. MA, SM and RT have received clinical research fellowships from the Western Australia Cancer and Palliative Care Network (WACPCN). YCGL is an NHMRC Career Development Fellow and has received project grant funding from the NHMRC, New South Wales Dust Disease Board, Sir Charles Gairdner Research Advisory Committee, Institute for Respiratory Health and Cancer Council of Western Australia.

Competing interests YCGL and NAM have served on the advisory boards for CareFusion Inc. and Sequana Medical Ltd. DF-K has also served as a consultant for CareFusion Inc.

Ethics approval Sir Charles Gairdner Group Human Research Ethics Committee

Provenance and peer review Not commissioned; externally peer reviewed.

Open Access This is an Open Access article distributed in accordance with the Creative Commons Attribution Non Commercial (CC BY-NC 4.0) license, which permits others to distribute, remix, adapt, build upon this work noncommercially, and license their derivative works on different terms, provided the original work is properly cited and the use is non-commercial. See: http:// creativecommons.org/licenses/by-nc/4.0/

\section{REFERENCES}

1. Davies $\mathrm{H}$, Lee $\mathrm{YC}$. Management of malignant pleural effusions: questions that need answers. Curr Opin Pulm Med 2013;19: 374-9.

2. Clive A, Kahan B, Hooper C, et al. Predicting survival in malignant pleural effusion: development and validation of the LENT prognostic score. Thorax 2014;69:1098-104. 
3. Thomas $\mathrm{R}$, Francis $\mathrm{R}$, Davies $\mathrm{H}$, et al. Interventional therapies for malignant pleural effusions: the present and future. Respirology 2014;19:809-22.

4. Bethune N. Pleural poudrage. J Thorac Cardiovasc Surg 1935;1935:251-61.

5. Dresler C, Olak J, Derndon J, et al. Phase III intergroup study of talc poudrage vs talc slurry sclerosis for malignant pleural effusion. Chest 2005; 127:909-15

6. Fysh E, Tremblay A, Feller-Kopman D, et al. Clinical outcomes of indwelling pleural catheter-related pleural infections: an international multicenter study. Chest 2013;144:15997-602.

7. Maskell N, Lee YC, Gleeson F. Randomized trials describing lung inflammation after pleurodesis with talc of varying particle size. Am J Respir Crit Care Med 2004;170:377-82.

8. Davies $\mathrm{H}$, Mishra $\mathrm{E}$, Kahan $\mathrm{B}$, et al. Effect of an indwelling pleural catheter vs chest tube and talc pleurodesis for relieving dyspnoea in patients with malignant pleural effusion: the TIME2 randomized controlled trial. JAMA 2012;307:2383-9.

9. Fysh $\mathrm{E}$, Waterer $\mathrm{G}$, Kendall $\mathrm{P}$, et al. Indwelling pleural catheters reduce inpatient days over pleurodesis for malignant pleural effusion. Chest 2012;142:394-400.

10. Hunt B, Farivar A, Vallieres E, et al. Thoracoscopic talc versus tunneled pleural catheters for palliation of malignant pleura effusions. Ann Thorac Surg 2012.

11. Putnam J, Light R, Rodriguez $\mathrm{R}$, et al. A randomized comparison of indwelling pleural catheter and doxycyline pleurodesis in the management of malignant pleural effusions. Cancer 1999;86:1992-9.

12. Thomas R, Budgeon $\mathrm{C}$, Kuok $\mathrm{Y}$, et al. Catheter tract metastasis associated with indwelling pleural catheters. Chest 2014:146:557-62.
13. Boshuizen R, Thomas R, Lee YC. Use of indwelling pleural catheter for management of malignant pleural effusions: the advantages. Curr Respir Care Rep 2013;2:93-9.

14. Tremblay A, Mason C, Michaud G. Use of tunnelled catheters for malignant pleural effusions in patients fit for pleurodesis. Eur Respir J 2007;30:759-62.

15. VanMeter M, McKee K, Kohlwes R. Efficacy and safety of tunnelled pleural catheters in adults with malignant pleural effusions: a systematic review. J Gen Intern Med 2011;26:70-6.

16. Fysh $\mathrm{E}$, Thomas $\mathrm{R}$, Read $\mathrm{C}$, et al. Protocol of the Australasian Malignant Pleural Effusion (AMPLE) trial: a multicentre randomised study comparing indwelling pleural catheter versus talc pleurodesis. BMJ Open 2014;4:e006757.

17. Mishra E, Corcoran J, Hallifax R, et al. Defining the minimal important difference for the visual analogue scale assessing dyspnea in patients with malignant pleural effusion. PLOS ONE 2015;10:e0123798

18. Norman $\mathrm{R}$, Cronin $\mathrm{P}$, Viney R. A pilot discrete choice experiment to explore preferences for EQ-5D-5L health states. Appl Health Econ Health Policy 2013;11:287-98.

19. Tremblay A, Michaud G. Single-center experience with 250 tunnelled pleural catheter insertions for malignant pleural effusion. Chest 2006;129:362-8.

20. Herdman M, Gudex C, Lloyd A, et al. Development and preliminary testing of the new five-level version of EQ-5D (EQ-5D-5L). Qual Life Res 2011;20:1727-36.

21. EuroQoLGroup. Euro-QoL-a new facility for the measurement of health-related quality of life. Health Policy 1990;16:199-208.

22. Pickard $\mathrm{A}$, Wilke $\mathrm{C}$, Lin $\mathrm{H}$, et al. Health utilities using the EQ-5D in studies of cancer. Pharmacoeconomics 2007;25: 365-84. 